\title{
The timing and type of nursing staff occupational injury and illness incidents, Veterans Health Administration, 2002-2011: a retrospective, population-based, descriptive analysis
}

\author{
Charles E. Welch, Kathleen M. McPhaul \\ Veterans Health Administration, Department of Veterans Affairs, Washington, DC, USA \\ Correspondence: Charles E. Welch. Address: Department of Veterans Affairs Central Office, Office of Public Health \\ (10P3), 810 Vermont Avenue, NW, Washington, DC 20420, USA. Telephone: 1-202-461-1038. Email: \\ charles.welch@va.gov.
}

Received: July 2, 2012

Accepted: August 7, 2012

Online Published: November 6, 2012

DOI : $10.5430 /$ jnep.v3n3p13

URL: http://dx.doi.org/10.5430/jnep.v3n3p13

\section{Abstract}

Background: While the majority of occupational injuries and illnesses that result in lost work days occur during typical day shift hours, the U.S. Bureau of Labor Statistics has noted that timing patterns often reflect the unique nature of different occupations. However, a literature search indicated that few studies have assessed the interplay between the timing and the type of nursing staff occupational injury and illness incidents in general, but especially so for those incidents that were recorded on an hourly basis.

Methods: This decade-long retrospective population-based study ascertained the timing of diverse types of reported occupational injury or illness incidents among Veterans Health Administration (VHA) nursing employees, who were classified between the nurse, practical nurse, and nursing assistant series. Using January 1, 2002 as the start date for the longitudinal surveillance of incidents, descriptive analyses included 55,424 VHA nursing employees who reported a total of 113,708 incidents between 2002 and 2011, of which 106,216 (93.4\%) were retained for this study, because they included both the specific time and the specific type of incident involved. Although nursing staff work shifts can vary widely, three "typical" 8-hour work shifts-that is to say, night shift: 23:01-07:00, day shift: 07:01-15:00, and evening shift: 15:01-23:00-were selected for summarizing study findings (i.e., for incidents that included both the specific time and the specific type of incident involved).

Results: Findings indicated that male nursing staff (accounting for $15.4 \%$ of the applicable occupational injury and illness incidents) reported a larger percentage of “Assaults” and "Lifting (Patient Care)" incidents, especially during the evening and the night shifts, whereas female nursing staff (accounting for $84.6 \%$ of the applicable incidents) reported a larger percentage of "Slips, Trips, and Falls" incidents, but these were more likely to occur during the beginning and the end of each shift. Findings also indicated that, regardless of gender, "Assaults” and "Lifting (Patient Care)" incidents were more commonly reported during the evening and the night shifts, as compared with the day shift, between all three nursing occupations (nurse, practical nurse, and nursing assistant). "Slips, Trips, and Falls" incidents were more commonly reported during the beginning and end of each shift, between all three nursing occupations. 
Conclusions: Staffing patterns and nursing staff working conditions are risk factors for occupational injuries and illnesses. Findings suggest that more attention is needed for ascertaining the potential role and functioning of targeted injury prevention training initiatives with respect to the timing and potential likelihood of selected types of nursing staff occupational injury or illness incidents.

\section{Key words}

Nursing staff, Timing of occupational injury incidents, Types of occupational injury incidents, Assaults/prevention \& control, Statistics \& numeric data

\section{Introduction}

While the majority of occupational injuries and illnesses that result in lost work days occur during typical day shift hours, the U.S. Bureau of Labor Statistics (BLS) has noted that timing patterns often reflect the unique nature of different occupations ${ }^{[1]}$. For the category of "nursing aides, orderlies, and attendants", BLS staff found that, during 2002, the highest percentage of occupational injuries and illnesses that resulted in lost work time occurred during the 10:01 a.m. to 12:00 noon (10:01 to 12:00) time period, whereas the lowest percentage occurred during the 2:01 a.m. to 4:00 a.m. (02:01 to $04: 00)$ time period.

However, a literature search indicated that few studies have assessed the interplay between the timing and the type of nursing staff occupational injury and illness incidents in general, but especially so for those incidents that were recorded on an hourly basis. For example, a two-year descriptive study that was conducted at a single, 497-bed hospital found that most occupational injuries of nursing and therapy personnel occurred between 9:30 a.m. and 11:30 a.m. (09:30 to 11:30) time period ${ }^{[2]}$.

Other studies have examined the association of shift type, number of work hours, and mandatory overtime with occupational injuries. For example, a seven-year retrospective analysis of workers compensation claims in Oregon found that healthcare workers sustained more injuries during the evening and nights shifts ${ }^{[3]}$. A longitudinal study of 2,617 registered nurses found that musculoskeletal injuries were adversely associated with extended work schedules ${ }^{[4]}$.

Recent comparisons of long-term historical BLS data trends for the private sector (i.e., for occupational injuries and illnesses that resulted in lost work time) and VHA data (i.e., for all reported "no-fault" occupational injuries and illnesses incidents, regardless of outcome) found similar overall percentage distributions across the six (four-hours each) daily time periods of the incidents that were routinely reported by BLS.

VHA staff conducted a comparative longitudinal analysis (unpublished; dated, December 19, 2011) of VHA and BLS data from 2002 through 2010. VHA staff found that 36.2 percent of the BLS sampled private sector occupational injury and illness incidents (with time of day reported, and that resulted in lost work time) occurred during the 8:01 a.m. and noon (08:01 to 12:00) time period, as compared with 36.7 percent of all reported VHA occupational injury and illness incidents (with time of day reported, but regardless of outcome) for VHA's healthcare system as a whole.

In a similar manner, from 2002 through 2010, the percentage distributions for three major types of occupational injury or illness incidents - i.e., assaults, over-exertion in lifting (as compared with the lifting of patients within VHA), and slips, trips, and falls were similar for BLS - i.e., 38.2 percent of the sampled private sector incidents that resulted in lost work time - and for all employees within VHA’s healthcare system as a whole (i.e., 37.0 percent of all reported incidents).

The purpose of this decade-long retrospective population-based study was to conduct a detailed assessment (i.e., on an hourly basis) of the timing of diverse types of reported occupational injury or illness incidents among those VHA nursing staff who were classified between the nurse, practical nurse, and nursing assistant series. One can posit that staffing patterns, scope and type of job duties, and other environmental conditions may vary substantially during the course of a 
day, and thus may have differential impacts on the potential risk exposures of diverse nursing staff for diverse types of occupational injury or illness incidents. Accordingly, this study seeks to determine whether timing patterns are associated with the types of occupational injury and illness incidents that diverse nursing staff occupations are likely to encounter, on an hourly basis. In turn, findings from this study should help to more fully inform VHA's development of targeted workplace injury prevention initiatives for its nursing staff employees.

\section{Subjects and methods}

Spanning the fifty States, American Samoa, Guam, the Philippine Islands, Puerto Rico, and the Virgin Islands, VHA is a complex Veteran-centric, vertically-integrated, healthcare system. As of October 2011, for example, VHA encompassed approximately 1,600 healthcare delivery sites, including 152 medical centers, 133 Community Living Centers (nursing homes), 293 Vet Centers, and 965 community-based outpatient clinics ${ }^{[5]}$.

Participants for this retrospective, population-based study were those VHA field-based nursing staff, who were classified (at the date and time of the reported incident) between three Office of Personnel Management series: nurses (610), practical nurses (620), and nursing assistants $(621)^{[6]}$.

Using January 1, 2002 as the start date for the 10-year longitudinal surveillance of incidents, descriptive analyses included 55,424 VHA nursing employees who reported a total of 113,708 incidents between 2002 and 2011, of which 106,216 incidents $(93.4 \%)$ were retained for this study, because they included both the specific time and specific type of the incident involved.

Virtually all (98.3\%) of the retained incidents were occupational injuries (i.e., as compared with occupational illnesses). Female nursing staff accounted for $84.6 \%$ of these applicably coded incidents.

\subsection{Data source}

VHA developed the Automated Safety Incident Surveillance and Tracking System (ASISTS) for comprehensive "no-fault" occupational injury and illness incident reporting at the local medical facility level. Injured staff, their supervisors, and safety personnel enter incident data into the local facility's computerized ASISTS database. Any entry may trigger a detailed investigation, although not necessarily a potentially compensable claim. After the incident reporting data are identified, investigated, coded/described, and "closed" at the local medical facility level, these cases are subsequently transmitted to a centralized VHA data repository in Austin, Texas- i.e., the Austin Information Technology Center [AITC]).

Subject to certain privacy restrictions, selected AITC ASISTS incident data are updated (typically on a monthly basis) and made accessible to all VA staff on both web-based (for the basic end user) and professional (interactive) desktop platforms.

Data for this study included all applicable 2002-2011 ASISTS cases that had been transmitted to Austin, Texas, as of June 8, 2012.

\subsection{Data limitations}

As an administrative database, changes in diagnostic and pathologic criteria, methods, and classifications over time can influence the accuracy of ASISTS incident data. As with most administrative databases, one may also encounter local facility issues regarding the under-reporting of work-related injuries in general ${ }^{[7,8]}$. 


\subsection{Methods}

Outcome measures were percentage distributions of type of incidents, by hour of the day, as differentiated by gender or by nursing staff occupation.

Types of incidents included:

- Assaults,

- Cumulative Trauma (e.g., carpal tunnel syndrome),

- Environmental/Exposure (including environmental toxic exposure, exposure to body fluids or splashes, latex reaction or allergy, and tuberculosis skin test conversions),

- $\quad$ Lifting (Non-Patient Care),

- $\quad$ Lifting (Patient Care) (e.g., repositioning of patients),

- Punctures (e.g., hollow bore needle sticks, sharps exposure, and suture needle sticks),

- Slips, Trips, and Falls,

- Other (e.g., material handling, struck by/against, and not elsewhere classified/other).

\subsection{Statistical analysis}

Data were analyzed using PASW-SPSS Statistics 18.

Given that these data were administrative in nature and endogenous only for the population of VHA medical facilities, no "inferential" statistical confidence intervals (or other statistical inferences) were provided for comparisons with nursing staff organizations that are external to VHA. Instead, all data summaries were expected to be interpreted as being descriptive (informative) only for VHA in their nature.

\section{Results}

Although nursing staff work shifts can vary widely, three "typical" 8-hour work shifts-that is to say, night shift: 23:01-07:00, day shift: 07:01-15:00, and evening shift: 15:01-23:00-were constructed for summarizing study findings (i.e., for incidents that included both the specific time and the specific type of incident involved).

Across 10 years (2002-2011), “Assaults” (11.4\%), “Lifting (Patient Care)” (22.5\%), and “Slips, Trips, and Falls” (18.4\%) accounted for over half (52.3\%) of the applicable VHA nursing staff occupational injury and illness incidents (i.e., with time of day recorded, but regardless of gender or type of occupation). The largest remaining single type of reported incident was the miscellaneous "Other" (27.4\%) category, followed by "Puncture" (9.7\%), "Environmental/Exposure (8.0\%), “Cumulative Trauma” (1.4\%), and "Lifting (Non-Patient Care)” (1.3\%) (see Table 1).

Detailed findings indicated that male nursing staff (accounting for $15.4 \%$ of the applicable occupational injury and illness incidents) reported a larger percentage of "Assaults" and "Lifting (Patient Care)" incidents, especially during the evening and the night shifts, whereas female nursing staff (accounting for $84.6 \%$ of the applicable incidents) reported a larger percentage of "Slips, Trips, and Falls" incidents, but these incidents were more likely to occur during the beginning and end of each shift. 
Table 1. Percentage Distribution of Timing and Type of Occupational Injury and Illness Incidents, by Gender: Veterans Health Administration Nursing Staff, 2002-2011

\begin{tabular}{|c|c|c|c|c|c|c|c|c|c|}
\hline Type of Incident & Daily & $\begin{array}{l}23: 01- \\
24: 00\end{array}$ & $\begin{array}{l}\text { 00:01- } \\
\text { 01:00 }\end{array}$ & $\begin{array}{l}\text { 01:01- } \\
02: 00\end{array}$ & $\begin{array}{l}\text { 02:01- } \\
\text { 03:00 }\end{array}$ & $\begin{array}{l}\text { 03:01- } \\
\text { 04:00 }\end{array}$ & $\begin{array}{l}\text { 04:01- } \\
\text { 05:00 }\end{array}$ & $\begin{array}{l}\text { 05:01- } \\
06: 00\end{array}$ & $\begin{array}{l}\text { 06:01- } \\
\text { 07:00 }\end{array}$ \\
\hline
\end{tabular}

Hour of the Day: Male Nursing Staff (11:01 PM to 7:00 AM, N=16,342)

\begin{tabular}{|c|c|c|c|c|c|c|c|c|c|}
\hline Assault & 17.5 & 23.2 & 21.5 & 25.9 & 22.3 & 20.1 & 17.9 & 19.8 & 12.7 \\
\hline Cumulative Trauma & 1.1 & 2.1 & 0.7 & 0.3 & 0.8 & 0.7 & 1.6 & 0.9 & 1.4 \\
\hline Environmental/Exposure & 8.4 & 7.6 & 7.2 & 10.3 & 6.7 & 9.1 & 9.2 & 7.3 & 8.2 \\
\hline Lifting (Non-Patient Care) & 1.4 & 0.0 & 0.7 & 1.4 & 0.0 & 0.7 & 0.7 & 0.7 & 2.4 \\
\hline Lifting (Patient Care) & 25.0 & 27.2 & 29.6 & 31.7 & 31.9 & 30.2 & 27.6 & 25.6 & 17.3 \\
\hline Puncture & 8.7 & 5.8 & 4.6 & 4.5 & 5.0 & 5.7 & 9.2 & 9.3 & 5.9 \\
\hline Slips, Trips, and Falls & 10.9 & 13.5 & 7.5 & 7.2 & 8.0 & 11.4 & 10.8 & 14.8 & 25.3 \\
\hline Other & 27.1 & 20.5 & 28.3 & 18.6 & 25.2 & 22.1 & 23.0 & 21.7 & 26.8 \\
\hline
\end{tabular}

\section{Hour of the Day: Female Nursing Staff (11:01 PM to 7:00 AM, N=89,874)}

$\begin{array}{llllllllll}\text { Assault } & 10.3 & 16.1 & 15.7 & 17.2 & 13.4 & 14.5 & 13.9 & 11.8 & 5.3 \\ \text { Cumulative Trauma } & 1.5 & 1.3 & 1.4 & 0.8 & 1.2 & 0.9 & 1.3 & 1.3 & 2.5 \\ \text { Environmental/Exposure } & 7.9 & 7.9 & 6.4 & 8.5 & 7.1 & 7.1 & 7.0 & 6.4 & 8.0 \\ \text { Lifting (Non-Patient Care) } & 1.3 & 1.2 & 1.2 & 1.1 & 0.8 & 1.0 & 0.5 & 1.1 & 1.5 \\ \text { Lifting (Patient Care) } & 22.0 & 21.9 & 31.5 & 30.8 & 31.1 & 31.5 & 27.9 & 21.3 & 10.4 \\ \text { Puncture } & 9.8 & 4.4 & 6.0 & 5.9 & 7.4 & 9.1 & 9.3 & 10.0 & 5.1 \\ \text { Slips, Trips, and Falls } & 19.7 & 23.1 & 14.2 & 13.6 & 14.3 & 14.1 & 18.5 & 25.7 & 42.1 \\ \text { Other } & 27.4 & 24.1 & 23.7 & 22.2 & 24.7 & 21.8 & 21.6 & 22.4 & 25.1\end{array}$

Hour of the Day: All Nursing Staff (11:01 PM to 7:00 AM, N =106,216)

\begin{tabular}{|c|c|c|c|c|c|c|c|c|c|}
\hline Assault & 11.4 & 13.3 & 17.3 & 16.7 & 18.8 & 14.9 & 15.4 & 14.5 & 12.9 \\
\hline Cumulative Trauma & 1.4 & 1.3 & 1.5 & 1.2 & 0.7 & 1.1 & 0.9 & 1.4 & 1.2 \\
\hline Environmental/Exposure & 8.0 & 7.2 & 7.9 & 6.5 & 8.8 & 7.0 & 7.4 & 7.3 & 6.5 \\
\hline Lifting (Non-Patient Care) & 1.3 & 1.1 & 1.0 & 1.1 & 1.2 & 0.6 & 1.0 & 0.6 & 1.0 \\
\hline Lifting (Patient Care) & 22.5 & 19.9 & 22.8 & 31.2 & 31.0 & 31.2 & 31.3 & 27.9 & 21.9 \\
\hline Puncture & 9.7 & 7.5 & 4.6 & 5.7 & 5.6 & 7.0 & 8.5 & 9.3 & 9.9 \\
\hline Slips, Trips, and Falls & 18.4 & 26.5 & 21.5 & 13.1 & 12.4 & 13.2 & 13.7 & 17.3 & 24.2 \\
\hline Other & 27.4 & 23.3 & 23.5 & 24.5 & 21.5 & 24.8 & 21.8 & 21.8 & 22.3 \\
\hline
\end{tabular}


Table 1. (Continued)

\begin{tabular}{llllllllll}
\hline \multirow{2}{*}{ Type of Incident } & \multirow{3}{*}{ Daily } & $07: 01-$ & $08: 01-$ & $09: 01-$ & $10: 01-$ & $11: 01-$ & $\begin{array}{l}12: 01- \\
13: 01-\end{array}$ & $\begin{array}{l}14: 01- \\
14: 00\end{array}$ \\
\end{tabular}

Hour of the Day: Male Nursing Staff (7:01 AM to 3:00 PM, N=16,342)

\begin{tabular}{|c|c|c|c|c|c|c|c|c|c|}
\hline Assault & 17.5 & 14.8 & 13.0 & 13.8 & 12.0 & 12.3 & 13.6 & 12.9 & 18.9 \\
\hline Cumulative Trauma & 1.1 & 1.1 & 1.3 & 0.9 & 1.1 & 0.9 & 1.5 & 1.1 & 1.3 \\
\hline Environmental/Exposure & 8.4 & 7.3 & 7.5 & 9.4 & 8.6 & 8.7 & 9.2 & 8.4 & 9.0 \\
\hline Lifting (Non-Patient Care) & 1.4 & 2.6 & 1.9 & 1.7 & 1.4 & 0.9 & 2.0 & 1.9 & 1.0 \\
\hline Lifting (Patient Care) & 25.0 & 22.7 & 26.4 & 24.7 & 23.7 & 23.1 & 25.0 & 25.7 & 22.0 \\
\hline Puncture & 8.7 & 8.8 & 9.1 & 9.7 & 13.0 & 12.5 & 9.7 & 10.3 & 8.6 \\
\hline Slips, Trips, and Falls & 10.9 & 11.2 & 9.6 & 11.4 & 11.0 & 10.2 & 9.1 & 8.9 & 12.5 \\
\hline Other & 27.1 & 31.5 & 31.1 & 28.4 & 29.2 & 31.3 & 30.0 & 30.8 & 26.7 \\
\hline \multicolumn{10}{|c|}{ Hour of the Day: Female Nursing Staff (7:01 AM to 3:00 PM, N=89,874) } \\
\hline Assault & 10.3 & 8.9 & 7.9 & 8.0 & 7.5 & 8.0 & 7.5 & 8.7 & 7.9 \\
\hline Cumulative Trauma & 1.5 & 1.6 & 1.6 & 1.1 & 1.4 & 1.5 & 1.8 & 1.6 & 2.0 \\
\hline Environmental/Exposure & 7.9 & 6.8 & 8.8 & 8.7 & 8.8 & 7.7 & 8.7 & 8.6 & 8.5 \\
\hline Lifting (Non-Patient Care) & 1.3 & 1.6 & 1.5 & 1.3 & 1.2 & 1.6 & 1.5 & 1.7 & 1.9 \\
\hline Lifting (Patient Care) & 22.0 & 18.8 & 23.1 & 23.8 & 19.9 & 20.2 & 23.7 & 21.8 & 18.6 \\
\hline Puncture & 9.8 & 9.4 & 10.8 & 11.6 & 13.6 & 11.3 & 9.2 & 10.6 & 9.4 \\
\hline Slips, Trips, and Falls & 19.7 & 22.7 & 17.2 & 16.6 & 17.8 & 19.5 & 16.9 & 16.1 & 22.0 \\
\hline Other & 27.4 & 30.0 & 29.1 & 28.9 & 29.8 & 28.5 & 30.8 & 30.8 & 29.8 \\
\hline
\end{tabular}

Hour of the Day: All Nursing Staff (7:01 AM to 3:00 PM, N=106,216)

$\begin{array}{lcccccccccc}\text { Assault } & 11.4 & 6.2 & 9.8 & 8.6 & 8.9 & 8.1 & 8.8 & 8.4 & 9.4 \\ \text { Cumulative Trauma } & 1.4 & 2.3 & 1.6 & 1.5 & 1.1 & 1.4 & 1.4 & 1.7 & 1.5 \\ \text { Environmental/Exposure } & 8.0 & 8.0 & 6.9 & 8.6 & 8.8 & 8.8 & 7.9 & 8.8 & 8.6 \\ \text { Lifting (Non-Patient Care) } & 1.3 & 1.6 & 1.7 & 1.6 & 1.4 & 1.2 & 1.5 & 1.6 & 1.7 \\ \text { Lifting (Patient Care) } & 22.5 & 11.3 & 19.4 & 23.6 & 24.0 & 20.4 & 20.9 & 23.9 & 22.3 \\ \text { Puncture } & 9.7 & 5.2 & 9.3 & 10.5 & 11.3 & 13.5 & 11.6 & 9.3 & 10.6 \\ \text { Slips, Trips, and Falls } & 18.4 & 40.0 & 21.1 & 16.2 & 15.8 & 16.9 & 18.5 & 15.8 & 15.0 \\ \text { Other } & 27.4 & 25.3 & 30.2 & 29.4 & 28.8 & 29.8 & 29.3 & 30.7 & 30.8\end{array}$


Table 1. (Continued)

\begin{tabular}{|c|c|c|c|c|c|c|c|c|c|}
\hline Type of Incident & Daily & $\begin{array}{l}15: 01- \\
16: 00\end{array}$ & $\begin{array}{l}16: 01- \\
17: 00\end{array}$ & $\begin{array}{l}\text { 17:01- } \\
18: 00\end{array}$ & $\begin{array}{l}\text { 18:01- } \\
19: 00\end{array}$ & $\begin{array}{l}\text { 19:01- } \\
20: 00\end{array}$ & $\begin{array}{l}\text { 20:01- } \\
\text { 21:00 }\end{array}$ & $\begin{array}{l}\text { 21:01- } \\
22: 00\end{array}$ & $\begin{array}{l}\text { 22:01- } \\
\text { 23:00 }\end{array}$ \\
\hline
\end{tabular}

Hour of the Day: Male Nursing Staff (3:01 PM to 11:00 PM, N = 16,342)

\begin{tabular}{|c|c|c|c|c|c|c|c|c|c|}
\hline Assault & 17.5 & 22.5 & 20.7 & 26.1 & 20.1 & 22.4 & 22.9 & 27.0 & 20.6 \\
\hline Cumulative Trauma & 1.1 & 1.0 & 1.3 & 0.9 & 0.8 & 0.6 & 0.9 & 1.1 & 1.4 \\
\hline Environmental/Exposure & 8.4 & 8.1 & 7.2 & 9.1 & 9.3 & 7.8 & 7.5 & 10.0 & 5.7 \\
\hline Lifting (Non-Patient Care) & 1.4 & 1.3 & 1.5 & 1.1 & 1.5 & 1.4 & 0.2 & 0.9 & 1.0 \\
\hline Lifting (Patient Care) & 25.0 & 23.7 & 23.2 & 26.9 & 29.0 & 25.5 & 28.7 & 23.9 & 24.7 \\
\hline Puncture & 8.7 & 8.2 & 10.1 & 5.8 & 5.9 & 7.2 & 10.8 & 6.9 & 6.1 \\
\hline Slips, Trips, and Falls & 10.9 & 11.0 & 7.8 & 7.0 & 9.1 & 8.4 & 8.0 & 6.3 & 16.9 \\
\hline Other & 27.1 & 24.1 & 28.3 & 23.2 & 24.3 & 26.7 & 21.0 & 23.9 & 23.6 \\
\hline
\end{tabular}

Hour of the Day: Female Nursing Staff (3:01 PM to 11:00 PM, N=89,874)

$\begin{array}{lccccccccc}\text { Assault } & 10.3 & 11.4 & 14.2 & 15.1 & 15.0 & 15.8 & 14.5 & 17.4 & 11.8 \\ \text { Cumulative Trauma } & 1.5 & 1.3 & 1.0 & 1.0 & 1.4 & 1.0 & 0.8 & 1.2 & 1.3 \\ \text { Environmental/Exposure } & 7.9 & 7.0 & 7.0 & 7.8 & 8.2 & 7.2 & 7.8 & 7.4 & 7.5 \\ \text { Lifting (Non-Patient Care) } & 1.3 & 1.3 & 0.9 & 0.8 & 1.3 & 1.0 & 1.1 & 0.8 & 1.1 \\ \text { Lifting (Patient Care) } & 22.0 & 18.5 & 22.8 & 26.0 & 25.9 & 26.2 & 24.8 & 24.9 & 19.0 \\ \text { Puncture } & 9.8 & 9.6 & 12.9 & 8.7 & 6.1 & 10.0 & 13.3 & 10.5 & 7.8 \\ \text { Slips, Trips, and Falls } & 19.7 & 24.0 & 15.1 & 14.4 & 15.9 & 13.1 & 13.1 & 13.5 & 28.4 \\ \text { Other } & 27.4 & 27.0 & 26.1 & 26.2 & 26.3 & 25.7 & 24.7 & 24.3 & 23.2\end{array}$

Hour of the Day: All Nursing Staff (3:01 PM to 11:00 PM, $N=106,216)$

\begin{tabular}{lccccccccc} 
Assault & 11.4 & 9.8 & 13.2 & 15.3 & 17.1 & 15.9 & 17.1 & 15.9 & 19.2 \\
Cumulative Trauma & 1.4 & 1.9 & 1.3 & 1.1 & 1.0 & 1.3 & 0.9 & 0.8 & 1.2 \\
Environmental/Exposure & 8.0 & 8.6 & 7.2 & 7.0 & 8.0 & 8.4 & 7.4 & 7.8 & 7.9 \\
Lifting (Non-Patient Care) & 1.3 & 1.7 & 1.3 & 1.0 & 0.9 & 1.3 & 1.0 & 0.9 & 0.8 \\
Lifting (Patient Care) & 22.5 & 19.2 & 19.3 & 22.8 & 26.1 & 26.5 & 26.0 & 25.4 & 24.7 \\
Puncture & 9.7 & 9.3 & 9.4 & 12.4 & 8.2 & 6.0 & 9.5 & 12.9 & 9.8 \\
Slips, Trips, and Falls & 18.4 & 20.4 & 21.8 & 13.9 & 13.1 & 14.7 & 12.2 & 12.2 & 12.2 \\
Other & 27.4 & 29.2 & 26.5 & 26.5 & 25.6 & 26.0 & 25.9 & 24.1 & 24.2 \\
\hline
\end{tabular}


Notably a study of workers compensation claims in West Virginia during 1997-1999 found that the healthcare sector sustained the bulk of assault injuries in West Virginia. Although the majority of healthcare-sector employees were female, the risk of assault injuries was apparently higher for male employees ${ }^{[9]}$.

Given their limited size as a proportion of the total VHA nursing staff population, all applicable incidents for male nursing staff were subsequently combined with those for female nursing staff, to support a statistically more robust descriptive analysis of the types of incidents, as differentiated on an hourly basis, between three nursing staff occupations (nurse, practical nurse, and nursing assistant).

Findings indicated that, regardless of gender, "Assaults" and "Lifting (Patient Care)" incidents were more commonly reported during the evening and the night shifts, as compared with the day shift, across all three nursing occupations. "Slips, Trips, and Falls" incidents were more commonly reported during the beginning and the end of each shift, across all three nursing occupations (see Table 2).

Table 2. Percentage Distribution of Timing and Type of Occupational Injury and Illness Incidents, by Nursing Occupation: Veterans Health Administration Nursing Staff, 2002-2011

\begin{tabular}{|c|c|c|c|c|c|c|c|c|c|}
\hline Type of Incident & Daily & $\begin{array}{l}23: 01- \\
24: 00\end{array}$ & $\begin{array}{l}\text { 00:01- } \\
\text { 01:00 }\end{array}$ & $\begin{array}{l}\text { 01:01- } \\
\text { 02:00 }\end{array}$ & $\begin{array}{l}\text { 02:01- } \\
\text { 03:00 }\end{array}$ & $\begin{array}{l}\text { 03:01- } \\
\text { 04:00 }\end{array}$ & $\begin{array}{l}\text { 04:01- } \\
\text { 05:00 }\end{array}$ & $\begin{array}{l}\text { 05:01- } \\
06: 00\end{array}$ & $\begin{array}{l}\text { 06:01- } \\
07: 00\end{array}$ \\
\hline \multicolumn{10}{|c|}{ Hour of the Day: Nurses (11:01 PM to 7:00 AM, $N=57,071)$} \\
\hline Assault & 7.7 & 9.1 & 14.3 & 14.8 & 14.4 & 11.2 & 12.9 & 11.0 & 7.2 \\
\hline Cumulative Trauma & 1.5 & 1.6 & 1.6 & 1.2 & 1.2 & 1.2 & 1.6 & 1.2 & 1.4 \\
\hline $\begin{array}{l}\text { Environmental/ } \\
\text { Exposure }\end{array}$ & 9.7 & 7.9 & 9.7 & 8.6 & 10.3 & 9.1 & 8.3 & 8.8 & 8.6 \\
\hline Lifting (Non-Patient Care) & 1.4 & 0.8 & 0.8 & 1.2 & 1.7 & 1.0 & 1.2 & 0.9 & 1.1 \\
\hline Lifting (Patient Care) & 19.6 & 19.5 & 23.5 & 28.3 & 26.8 & 29.6 & 27.8 & 24.3 & 17.4 \\
\hline Puncture & 12.6 & 11.4 & 6.7 & 8.2 & 8.7 & 10.2 & 13.3 & 13.5 & 12.7 \\
\hline Slips, Trips, and Falls & 21.0 & 25.6 & 19.0 & 13.8 & 13.5 & 12.1 & 13.4 & 20.2 & 31.1 \\
\hline Other & 26.4 & 24.1 & 24.3 & 23.9 & 23.4 & 25.7 & 21.5 & 20.1 & 20.6 \\
\hline \multicolumn{10}{|c|}{ Hour of the Day: Practical Nurses (11:01 PM to 7:00 AM,N = 22,191) } \\
\hline Assault & 11.2 & 11.3 & 18.1 & 13.3 & 21.4 & 14.8 & 16.0 & 13.8 & 11.8 \\
\hline Cumulative Trauma & 1.4 & 1.1 & 0.8 & 0.9 & 0.3 & 2.0 & 0.0 & 2.2 & 0.7 \\
\hline Environmental/Exposure & 7.2 & 7.0 & 6.6 & 6.0 & 8.3 & 6.4 & 6.8 & 6.3 & 6.1 \\
\hline Lifting (Non-Patient Care) & 1.2 & 1.1 & 1.3 & 1.8 & 0.3 & 0.0 & 1.2 & 0.2 & 1.4 \\
\hline Lifting (Patient Care) & 22.6 & 21.5 & 19.9 & 32.2 & 34.9 & 33.2 & 38.5 & 27.0 & 23.0 \\
\hline Puncture & 10.5 & 6.7 & 2.4 & 4.2 & 4.9 & 4.8 & 4.7 & 9.4 & 14.5 \\
\hline Slips, Trips, and Falls & 17.5 & 28.0 & 25.7 & 14.2 & 11.6 & 14.8 & 13.9 & 17.4 & 21.8 \\
\hline Other & 28.4 & 23.4 & 25.2 & 27.4 & 18.3 & 24.0 & 18.9 & 23.7 & 20.7 \\
\hline
\end{tabular}


Table 2. (Continued)

\begin{tabular}{|c|c|c|c|c|c|c|c|c|c|}
\hline Type of Incident & Daily & $\begin{array}{l}23: 01- \\
24: 00\end{array}$ & $\begin{array}{l}\text { 00:01- } \\
\text { 01:00 }\end{array}$ & $\begin{array}{l}\text { 01:01- } \\
\text { 02:00 }\end{array}$ & $\begin{array}{l}\text { 02:01- } \\
\text { 03:00 }\end{array}$ & $\begin{array}{l}\text { 03:01- } \\
\text { 04:00 }\end{array}$ & $\begin{array}{l}\text { 04:01- } \\
\text { 05:00 }\end{array}$ & $\begin{array}{l}\text { 05:01- } \\
06: 00\end{array}$ & $\begin{array}{l}\text { 06:01- } \\
\text { 07:00 }\end{array}$ \\
\hline \multicolumn{10}{|c|}{ Hour of the Day: Nursing Assistants (11:01 PM to 7:00 AM, N=26,954) } \\
\hline Assault & 19.4 & 22.0 & 22.6 & 22.7 & 24.8 & 22.6 & 19.5 & 19.8 & 22.4 \\
\hline Cumulative Trauma & 1.2 & 1.0 & 1.7 & 1.6 & 0.0 & 0.5 & 0.2 & 1.1 & 1.3 \\
\hline Environmental/Exposure & 5.0 & 6.1 & 5.2 & 2.8 & 6.4 & 3.4 & 6.2 & 5.9 & 3.6 \\
\hline Lifting (Non-Patient Care) & 1.2 & 1.6 & 1.0 & 0.4 & 0.9 & 0.3 & 0.4 & 0.3 & 0.7 \\
\hline Lifting (Patient Care) & 28.5 & 19.4 & 23.2 & 35.9 & 35.7 & 33.2 & 32.8 & 33.1 & 28.3 \\
\hline Puncture & 2.7 & 1.2 & 2.1 & 2.0 & 0.7 & 2.1 & 2.5 & 3.4 & 2.4 \\
\hline Slips, Trips, and Falls & 13.5 & 26.9 & 23.4 & 10.8 & 11.2 & 14.5 & 14.1 & 13.3 & 15.1 \\
\hline Other & 28.5 & 21.8 & 20.7 & 23.7 & 20.4 & 23.4 & 24.3 & 23.1 & 26.1 \\
\hline \multicolumn{10}{|c|}{ Hour of the Day: Nurses (7:01 AM to 3:00 PM, $N=57,071)$} \\
\hline Assault & 7.7 & 3.7 & 5.8 & 5.4 & 6.2 & 5.1 & 5.6 & 5.7 & 6.7 \\
\hline Cumulative Trauma & 1.5 & 2.6 & 1.8 & 1.6 & 1.0 & 1.5 & 1.5 & 1.9 & 1.4 \\
\hline Environmental/Exposure & 9.7 & 8.9 & 8.3 & 10.8 & 10.7 & 10.4 & 9.3 & 10.4 & 9.9 \\
\hline Lifting (Non-Patient Care) & 1.4 & 1.8 & 1.8 & 1.8 & 1.3 & 1.4 & 1.6 & 1.7 & 1.6 \\
\hline Lifting (Patient Care) & 19.6 & 8.5 & 16.0 & 18.6 & 20.2 & 18.2 & 18.7 & 21.6 & 18.9 \\
\hline Puncture & 12.6 & 5.8 & 11.6 & 13.5 & 14.7 & 16.9 & 14.9 & 11.9 & 13.5 \\
\hline Slips, Trips, and Falls & 21.0 & 44.9 & 25.1 & 19.5 & 18.2 & 18.9 & 21.0 & 17.8 & 17.0 \\
\hline Other & 26.4 & 23.9 & 29.8 & 28.9 & 27.6 & 27.6 & 27.4 & 28.9 & 31.0 \\
\hline
\end{tabular}

Hour of the Day: Practical Nurses (7:01 AM to 3:00 PM, N = 22,191)

\begin{tabular}{|c|c|c|c|c|c|c|c|c|c|}
\hline Assault & 11.2 & 5.8 & 9.1 & 8.0 & 8.4 & 8.5 & 8.6 & 8.4 & 9.4 \\
\hline Cumulative Trauma & 1.4 & 2.5 & 1.3 & 1.9 & 1.2 & 0.9 & 1.3 & 1.8 & 2.0 \\
\hline Environmental/Exposure & 7.2 & 8.6 & 6.5 & 8.7 & 7.8 & 8.1 & 7.3 & 8.2 & 7.1 \\
\hline Lifting (Non-Patient Care) & 1.2 & 1.2 & 1.5 & 1.2 & 1.5 & 0.9 & 1.6 & 1.4 & 1.8 \\
\hline Lifting (Patient Care) & 22.6 & 11.3 & 19.0 & 25.3 & 22.1 & 20.2 & 21.9 & 23.2 & 23.5 \\
\hline Puncture & 10.5 & 7.0 & 10.7 & 11.9 & 12.8 & 14.3 & 11.1 & 9.0 & 10.4 \\
\hline Slips, Trips, and Falls & 17.5 & 38.3 & 18.7 & 13.8 & 14.8 & 15.3 & 16.4 & 15.4 & 15.2 \\
\hline Other & 28.4 & 25.4 & 33.2 & 29.2 & 31.3 & 31.9 & 31.8 & 32.6 & 30.7 \\
\hline
\end{tabular}


Table 2. (Continued)

\begin{tabular}{|c|c|c|c|c|c|c|c|c|c|}
\hline Type of Incident & Daily & $\begin{array}{l}23: 01- \\
24: 00\end{array}$ & $\begin{array}{l}\text { 00:01- } \\
\text { 01:00 }\end{array}$ & $\begin{array}{l}\text { 01:01- } \\
\text { 02:00 }\end{array}$ & $\begin{array}{l}\text { 02:01- } \\
\text { 03:00 }\end{array}$ & $\begin{array}{l}\text { 03:01- } \\
\text { 04:00 }\end{array}$ & $\begin{array}{l}\text { 04:01- } \\
\text { 05:00 }\end{array}$ & $\begin{array}{l}\text { 05:01- } \\
\text { 06:00 }\end{array}$ & $\begin{array}{l}\text { 06:01- } \\
\text { 07:00 }\end{array}$ \\
\hline \multicolumn{10}{|c|}{ Hour of the Day: Nursing Assistants (7:01 AM to 3:00 PM, N = 26,954) } \\
\hline Assault & 19.4 & 14.4 & 19.5 & 16.1 & 14.8 & 15.4 & 17.6 & 15.0 & 16.0 \\
\hline Cumulative Trauma & 1.2 & 1.4 & 1.3 & 0.9 & 1.2 & 1.5 & 1.3 & 1.3 & 1.5 \\
\hline Environmental/Exposure & 5.0 & 4.6 & 4.2 & 3.9 & 5.6 & 5.3 & 4.7 & 5.4 & 6.6 \\
\hline Lifting (Non-Patient Care) & 1.2 & 1.6 & 1.6 & 1.4 & 1.3 & 0.8 & 1.3 & 1.4 & 1.8 \\
\hline Lifting (Patient Care) & 28.5 & 19.7 & 27.6 & 32.6 & 33.4 & 26.4 & 26.0 & 29.9 & 29.9 \\
\hline Puncture & 2.7 & 1.4 & 2.8 & 3.1 & 2.8 & 4.1 & 3.3 & 3.1 & 3.6 \\
\hline Slips, Trips, and Falls & 13.5 & 27.2 & 14.2 & 11.3 & 11.5 & 13.1 & 13.5 & 10.8 & 10.1 \\
\hline Other & 28.5 & 29.6 & 28.7 & 30.6 & 29.3 & 33.4 & 32.4 & 33.1 & 30.5 \\
\hline \multicolumn{10}{|c|}{ Hour of the Day: Nurses (3:01 PM to 11:00 PM, N = 57,071) } \\
\hline Assault & 7.7 & 7.2 & 9.0 & 9.8 & 10.2 & 10.6 & 14.3 & 11.3 & 14.0 \\
\hline Cumulative Trauma & 1.5 & 2.0 & 1.4 & 0.9 & 1.1 & 1.3 & 0.6 & 0.8 & 1.5 \\
\hline Environmental/Exposure & 9.7 & 10.5 & 8.5 & 8.8 & 10.8 & 11.8 & 9.1 & 9.1 & 9.0 \\
\hline Lifting (Non-Patient Care) & 1.4 & 1.7 & 1.6 & 0.8 & 0.9 & 1.8 & 1.1 & 1.0 & 0.9 \\
\hline Lifting (Patient Care) & 19.6 & 17.4 & 18.2 & 22.1 & 25.1 & 23.3 & 22.5 & 23.2 & 23.5 \\
\hline Puncture & 12.6 & 11.8 & 11.2 & 17.2 & 12.6 & 8.3 & 13.7 & 17.1 & 13.2 \\
\hline Slips, Trips, and Falls & 21.0 & 21.7 & 25.4 & 16.1 & 14.5 & 17.7 & 13.8 & 12.9 & 14.5 \\
\hline Other & 26.4 & 27.6 & 24.8 & 24.3 & 24.8 & 25.1 & 24.9 & 24.7 & 23.3 \\
\hline \multicolumn{10}{|c|}{ Hour of the Day: Practical Nurses (3:01 PM to 11:00 PM, N = 22,191) } \\
\hline Assault & 11.2 & 8.9 & 13.7 & 17.0 & 19.5 & 15.7 & 15.3 & 14.2 & 20.0 \\
\hline Cumulative Trauma & 1.4 & 1.7 & 1.5 & 1.1 & 0.9 & 1.1 & 1.3 & 0.8 & 1.2 \\
\hline Environmental/Exposure & 7.2 & 7.4 & 6.1 & 4.8 & 6.2 & 6.1 & 6.4 & 7.5 & 8.0 \\
\hline Lifting (Non-Patient Care) & 1.2 & 1.6 & 0.3 & 1.1 & 0.9 & 1.1 & 0.4 & 0.8 & 0.8 \\
\hline Lifting (Patient Care) & 22.6 & 19.8 & 17.8 & 22.3 & 26.5 & 27.8 & 25.3 & 25.3 & 22.8 \\
\hline Puncture & 10.5 & 10.2 & 11.4 & 13.7 & 7.8 & 5.9 & 11.3 & 16.2 & 12.0 \\
\hline Slips, Trips, and Falls & 17.5 & 18.8 & 21.7 & 12.4 & 12.9 & 16.8 & 11.7 & 13.0 & 8.8 \\
\hline Other & 28.4 & 31.6 & 27.5 & 27.6 & 25.3 & 25.6 & 28.5 & 22.1 & 26.5 \\
\hline
\end{tabular}


Table 2. (Continued)

\begin{tabular}{|c|c|c|c|c|c|c|c|c|c|}
\hline Type of Incident & Daily & $\begin{array}{l}23: 01- \\
24: 00\end{array}$ & $\begin{array}{l}00: 01- \\
01: 00\end{array}$ & $\begin{array}{l}\text { 01:01- } \\
\text { 02:00 }\end{array}$ & $\begin{array}{l}02: 01- \\
03: 00\end{array}$ & $\begin{array}{l}\text { 03:01- } \\
\text { 04:00 }\end{array}$ & $\begin{array}{l}04: 01- \\
05: 00\end{array}$ & $\begin{array}{l}05: 01- \\
06: 00\end{array}$ & $\begin{array}{l}06: 01 \\
07: 00\end{array}$ \\
\hline \multicolumn{10}{|c|}{ Hour of the Day: Nursing Assistants (3:01 PM to 11:00 PM, N = 26,954) } \\
\hline Assault & 19.4 & 16.7 & 22.5 & 24.7 & 25.7 & 23.7 & 22.8 & 26.4 & 27.7 \\
\hline Cumulative Trauma & 1.2 & 1.6 & 0.8 & 1.4 & 0.9 & 1.3 & 1.1 & 0.8 & 0.6 \\
\hline Environmental/Exposure & 5.0 & 5.1 & 5.4 & 5.3 & 5.1 & 5.0 & 5.3 & 5.3 & 6.0 \\
\hline Lifting (Non-Patient Care) & 1.2 & 1.9 & 1.5 & 1.3 & 0.8 & 0.8 & 1.4 & 0.8 & 0.7 \\
\hline Lifting (Patient Care) & 28.5 & 22.8 & 23.1 & 24.7 & 27.5 & 30.0 & 32.1 & 29.9 & 28.1 \\
\hline Puncture & 2.7 & 2.4 & 3.5 & 2.1 & 1.9 & 2.9 & 1.6 & 1.8 & 2.3 \\
\hline Slips, Trips, and Falls & 13.5 & 18.6 & 13.7 & 10.6 & 11.0 & 8.8 & 10.0 & 10.2 & 10.6 \\
\hline Other & 28.5 & 31.0 & 29.5 & 29.9 & 27.1 & 27.5 & 25.8 & 24.7 & 23.9 \\
\hline
\end{tabular}

Given that these respective percentage distributions were calculated on an hourly basis, one can group these data across a wide array of shifts. However, it remains evident that nursing assistants bear the greatest burden of encountering the types of (potentially preventable) incidents that are commonly associated with potentially adverse outcomes - that is to say, “Assaults" and "Lifting (Patient Care)" (e.g., repositioning of patients).

In general, nursing assistants (at the date and time of the reported incident) were younger - i.e., an average age of 44.2 years, as compared with 47.9 years for nurses, and 45.8 years for practical nurses. They were more likely to be male - i.e., $22.1 \%$ of the nursing assistants were male, as compared with $13.3 \%$ of the nurses and $12.6 \%$ of the practical nurses.

However, they were less likely to have achieved higher educational attainment levels. For example, 3.5\% of nursing assistants had achieved a maximum educational attainment level of a Bachelor's degree (or higher), as compared with $56.3 \%$ of the nurses, and $4.3 \%$ of the practical nurses.

As noted above, BLS found that during 2002, for the category of "nursing aides, orderlies, and attendants," the highest percentage (15.1\%) of sampled private sector occupational injuries and illnesses that resulted in lost work time occurred during the 10:01 a.m. to 12:00 noon (10:01 to 12:00) time period, followed closely (14.3\%) by the 8:01 a.m. to 10:00 a.m. (08:01 to 10:00) time period, whereas the lowest percentage (2.5\%) occurred during the 2:01 a.m. to 4:00 a.m. (02:01 to 04:00) time period ${ }^{[1]}$.

For VA nursing assistants during 2002-2011, the highest percentage (14.5\%) of applicable occupational injury and illness incidents occurred during the 8:01 a.m. to 10:00 a.m. (08:01 to 10:00) time period, followed closely (13.9\%) by the 10:01 a.m. to 12:00 noon (10:01 to 12:00) time period, whereas the lowest percentage (3.1\%) occurred during the 2:01 a.m. to 4:00 a.m. (02:01 to 04:00) time period.

\section{Proportion of ASISTS injuries resulting in compensable claims}

The Department of Veterans Affairs (VA) (Workers Compensation Management Information System (WCMIS) collects and maintains Lost Work Time statistical data. Limited lost work time data (yes/no) were provided for VHA's weekly AITC ASISTS data tapes through approximately mid-2005. 
As noted in a prior comparative longitudinal analysis (unpublished; dated, December 19, 2011), from 2002 through 2010, VHA staff found that lost time cases account for an estimated $30.0 \%$ of all BLS recordable cases.

Within the current ASISTS analysis, for all applicable nursing staff (i.e., nurses, practical nurses, and nursing assistants) who received medical or compensation funding for VA workers compensation claims, $14.0 \%$ of the VHA male nursing staff and $13.4 \%$ of the VHA female nursing staff were coded as definitely having had lost work time. In contrast, $70.0 \%$ of the VHA male nursing staff and $70.1 \%$ of the VHA female nursing staff were coded as having had no lost work time. The remaining $16.0 \%$ of the VHA male nursing staff and $16.5 \%$ of the VHA female nursing staff were coded as being “unknown”.

Importantly, from 2001 through 2005, the timing of ASISTS cases with paid workers compensation claims differed little from the timing of all ASISTS cases.

- For all VHA male nursing staff, 16.6\% of all incidents occurred during the 23:01 through 07:00 time period, 50.9\% occurred during the 07:01 through 15:00 time period, and 32.5\% occurred during the 15:01 through 23:00 time period.

- For all VHA male nursing staff paid workers compensation claims, $17.3 \%$ of all incidents occurred during the 23:01 through 07:00 time period, 52.2\% occurred during the 07:01 through 15:00 time period, and 30.5\% occurred during the 15:01 through 23:00 time period.

- For all VHA female nursing staff, 16.5\% of all incidents occurred during the 23:01 through 07:00 time period, 56.0\% occurred during the 07:01 through 15:00 time period, and 27.5\% occurred during the 15:01 through 23:00 time period.

- For all VHA female nursing staff paid workers compensation claims, $17.5 \%$ of all incidents occurred during the 23:01 through 07:00 time period, 54.6\% occurred during the 07:01 through 15:00 time period, and 27.9\% occurred during the 15:01 through 23:00 time period.

As noted above, limited VA WCMIS workers compensation claims data (through June 6, 2006) were available for applicable VHA ASISTS cases that originated from 2001 through 2005. However, it should be clearly noted that the occurrence of paid workers compensation claims for diverse types of occupational injury or illness incidents may not correspond with their ultimate financial burden for VHA as a whole.

- For example, for VHA male nursing staff, as of June 6, 2006, “Assaults” accounted for $16.7 \%$ of all incident types (for claims) from 2001 through 2005, but accounted for only 9.1\% of VHA’s associated costs for workers compensation claims, whereas "Lifting (Patient Care)" accounted for only 25.2\% of all incident types, but accounted for $37.8 \%$ of VHA's associated costs.

- In a similar manner, for VHA male nursing staff, "Slips, Trips, and Falls” accounted for only $10.4 \%$ of all incident types, but accounted for $18.2 \%$ of VHA's associated costs for workers compensation claims, whereas "Puncture(s)" accounted for $9.1 \%$ of all incident types, but accounted for only $0.4 \%$ of VHA's associated costs.

- For VHA female nursing staff, as of June 6, 2006, “Assaults” accounted for 9.8\% of all incident types (for claims) from 2001 through 2005, but accounted for only 5.3\% of VHA's associated costs for workers compensation claims, whereas "Lifting (Patient Care)" accounted for only 23.2\% of all incident types, but accounted for 34.5\% of VHA’s associated costs. 
- In a similar manner, for VHA female nursing staff, Slips, Trips, and Falls” accounted for only $16.8 \%$ of all incident types, but accounted for $22.1 \%$ of VHA's associated costs for workers compensation claims, whereas "Puncture(s)" accounted for $9.7 \%$ of all incident types, but accounted for only $0.9 \%$ of VHA's associated costs.

\section{Limitations}

It should be clearly noted that although these study findings are population-based, they are limited in their interpretation for organizations that are external to VHA. Similarly, by aggregating the data across ten years, one cannot readily differentiate the impact of internal temporal trends associated with changes in VHA's administrative structures, its patient population, its occupational and its safety and health programs, or its diverse healthcare delivery models.

\section{Discussion}

Among the three nursing occupations in this study, the higher likelihood of "Assaults” and "Lifting (Patient Care)" incidents occurring during the evening and night shifts-especially for VHA male staff in general, and for VHA nursing assistants in particular-may reflect both staffing patterns and nursing staff working conditions. In a similar manner, the higher likelihood of "Slips, Trips, and Falls" incidents occurring during the beginning and the end of each shift may represent a complex interplay between local environmental factors and nursing staff demographics that contribute to potentially avoidable poor outcomes.

Evaluation of local interventions based on local facility exposure assessments and working conditions is needed to address the apparent increased risk of occupational injuries for nursing personnel at certain high-risk times of the healthcare daily work cycle.

It is well known that staffing patterns and nursing staff working conditions are risk factors for occupational injuries and illnesses. However, these findings suggest that more attention is needed for ascertaining the potential for targeted injury prevention programming directed toward the timing and potential likelihood of selected types of nursing staff occupational injury or illness incidents.

The VHA Office of Public Health Occupational Health Strategic Health Group (OHSHG) develops policies, training, safety tools and provides consultation to the field for occupational injury prevention. For example, two such initiatives, the VHA Safe Patient Handling (SPH) program and the Behavior Threat Management Program (BTMP) address injury prevention from patient lifting and patient assault ${ }^{[10]}$.

These findings suggest that VHA's Office of Nursing Services and VHA's Safety and Health Office should conduct a detailed hourly "exposure assessment" of the types of work performed across the twenty-four hour continuum during which healthcare is being delivered. Such an assessment may reveal patterns of exposure to physical demands (i.e., lifting and transferring patients), violence (agitated or frustrated patients/visitors), rushing or running (resulting in slips and trips), or exposure to sharps or blood. Safety resources such as mechanical lifts, more staff training and education, or increased security could be deployed during times of higher risk.

\section{Conclusion}

Study findings clearly support the BLS contention that timing patterns often reflect the unique nature of different occupations ${ }^{[1]}$. They also suggest that more attention is needed for ascertaining the potential role and functioning of targeted injury prevention training initiatives with respect to the timing and the potential likelihood of selected types of VHA nursing staff occupational injury or illness incidents. 


\section{References}

[1] U.S. Department of Labor, Bureau of Labor Statistics (BLS). Time of lost-workday injuries and illnesses, 2002- first results announced by BLS. [Publication No. USDL 04-2407, December 2, 2004]. Available from: http://www.bls.gov/iif/oshwc/osh/os/osnr0020.pdf

[2] Gropelli T, Corle K. Assessment of nurses' and therapists' occupational musculoskeletal injuries. Medsurg Nurs. 2011; 20: 297-303. PMid:22409113

[3] Horwitz IB, McCall BP. The impact of shift work on the risk and severity of injuries for hospital employees: an analysis using Oregon workers’ compensation data. Occup Med (Lond). 2004; 54: 556-563. PMid:15385648 http://dx.doi.org/10.1093/occmed/kqh093

[4] Trinkoff AM, Le R, Geiger-Brown J, Lipscomb J, Lang G. Longitudinal relationship of work hours, mandatory overtime, and on-call to musculoskeletal problems in nurses. Am J Ind Med. 2006; 49: 964-971. PMid:16691609 http://dx.doi.org/10.1002/ajim.20330

[5] U.S. Department of Veterans Affairs, Office of Communications. Facts about the Veterans Health Administration (VHA). October 6, 2011. Available from: http://www.patientsafety.gov/NEWS/NCPSBg/VHAFactSheet2011.pdf

[6] U.S. Office of Personnel Management. Handbook of Occupational Groups and Families [updated, May 2009]. Available from: http://www.opm.gov/ fedclass/gshbkocc.pdf

[7] Siddharthan K, Hodgson M, Rosenberg D, Haiduven D, Nelson A. Under-reporting of work-related musculoskeletal disorders in the Veterans Administration. Int J Health Care Qual Assur Inc Leadersh Health Serv. 2006; 19: 463-476. PMid:17100218

[8] Somani RK, Khowaja K. Workplace violence towards nurses: a reality from the Pakistani context. J Nurs Educ Practice. 2012; 2(3): 148-153. Available from: http://dx.doi.org/10.5430/jnep.v2n3p148

[9] Islam SS, Edla SR, Mujuru P, Doyle EJ, Ducatman AM. Risk factors for physical assault: State-managed workers' compensation experience. Am J Prev Med. 2003; 25: 31-37. http://dx.doi.org/10.1016/S0749-3797(03)00095-3

[10] Mohr DC, Warren N, Hodgson MJ, Drummond DJ. Assault rates and implementation of a workplace violence prevention program in the Veterans Health Care Administration. J Occup Environ Med. 2011; 53: 511-516. PMid:21555925

http://dx.doi.org/10.1097/JOM.0b013e31820d101e 